\title{
RELATIONS BETWEEN TRAVELLING WAVE SOLUTIONS OF QUASILINEAR PARABOLIC EQUATIONS
}

\author{
HANS ENGLER
}

ABstract. A transformation between travelling wave solutions of $v_{t}=v_{x x}+f(v)$ and $u_{t}=\left(D(u) u_{x}\right)_{x}+g(u)$ is given.

1. Introduction, assumptions, main result. In this note we study travelling wave solutions of the semilinear diffusion equations

$$
v_{t}(x, t)=v_{x x}(x, t)+f(v(x, t))
$$

and

$$
u_{t}(x, t)=\left(D(u(x, t)) \cdot u_{x}(x, t)\right)_{x}+g(u(x, t))
$$

on the real line. Here, subscripts denote differentiation with respect to the corresponding variable. Equations (1) and (2) arise in simple models for flame propagation and for the spread of biological populations (cf. $[4,6])$; in those applications, $u$ (resp. $v$ ) is a temperature or a density. In particular, (2) allows for density-dependent diffusion, as proposed in [5].

We look for solutions that "travel" to the right at constant speed $c>0$ without changing their shape, i.e. for solutions $v(x, t)=V(x-c t), u(x, t)=U(x-c t)$. Then (1) and (2) become

$$
\begin{gathered}
c \cdot V^{\prime}(\xi)+V^{\prime \prime}(\xi)=-f(V(\xi)), \\
c \cdot U^{\prime}(\xi)+\left(D(U(\xi)) \cdot U^{\prime}(\xi)\right)^{\prime}=-g(U(\xi)) .
\end{gathered}
$$

Solutions will be understood in the weak sense, i.e. $U$ (resp. $V$ ) should be absolutely continuous, $f(V)$ (resp. $D(U) \cdot U^{\prime}$ and $g(U)$ ) should be locally integrable, and $\left(1^{\prime}\right)$ (resp. (2')) should hold in the sense of distributions. Also, only monotone solutions $U, V$ will be discussed; we assume that $V(+\infty)=U(+\infty)=0, V(-\infty)=U(-\infty)=$ $1, V(0)=U(0)=\frac{1}{2}$, which can always be achieved by a simple transformation of variables, once $U$ (resp. $V$ ) is known to possess limits at $+\infty$ and $-\infty$. Concerning $f, g$, and $D$, we assume that

$$
\begin{gathered}
f, g \in C^{0}([0,1]) \cap C^{1}((0,1)), \\
f(0)=g(0)=f(1)=g(1)=0, \\
D \in C^{1}((0,1)), \quad D(r)>0 \quad \text { for all } r \in(0,1) .
\end{gathered}
$$

Received by the editors March 27, 1984.

1980 Mathematics Subject Classification. Primary 34C20, 35 U55.

(C)1985 American Mathematical Society $0002-9939 / 85 \$ 1.00+\$ .25$ per page 
The purpose of this note is to show that a wave solution $V$ of $\left(1^{\prime}\right)$ exists iff a wave solution $U$ of $\left(2^{\prime}\right)$ exists, if $f(r)=g(r) \cdot D(r)$. Both solutions have the same speed and are related through a transformation of coordinates which can be explicitly given as soon as one of the solutions is known. We also show that, in this case, $V$ (resp. $U$ ) can also be obtained as a wave solution (with the same speed) of "conservation-law" type equations

$$
\begin{gathered}
v_{t}-v_{x x}=k(v)_{x}, \\
u_{t}-\left(D(u) u_{x}\right)_{x}=k(u)_{x},
\end{gathered}
$$

this time with the same $k(\cdot)$. Wave solutions of $\left(1^{\prime \prime}\right)$ and $\left(2^{\prime \prime}\right)$ can be obtained explicitly, since the resulting first order equation for $U, V$ is autonomous; this allows us to find (in fact, retrieve well-known) special solutions for certain classes of (1) and (2). The function $k(\cdot)$ is related to the function $p=P(q)$ whose graph is given in the $(q, p)$ phase plane by the trajectory $\left(V(\xi), V^{\prime}(\xi)\right)$, through the shear transformation $k(q)=-c q-P(q)$, which allows us to use many of the general results on $P$ given in [2].

Our main result is

THEOREM. Fix $c>0$, assume (3)-(5), and

$$
f(r)=g(r) \cdot D(r) \quad \text { for } 0<r<1 .
$$

The following statements are equivalent:

(i) There is a travelling wave solution $V_{1}$ of (1) with speed $c$ and the properties given above.

(ii) There is a travelling wave solution $V_{2}$ of $\left(1^{\prime \prime}\right)$ with speed $c$ and the properties given above, and

$$
\begin{gathered}
\frac{d}{d r} k(r) \cdot(c \cdot r+k(r))=-f(r) \quad \text { for } 0<r<1, \\
k(0)=0, \quad k(1)=-c, \quad k(r)+c r>0 .
\end{gathered}
$$

(iii) There is a travelling wave solution $W_{1}$ of (2) with speed $c$ and properties as above.

(iv) There is a travelling wave solution $W_{2}$ of (2") with speed $c$ and properties as above, and $k$ satisfies (7).

Moreover, $V_{1}=V_{2}, W_{1}=W_{2}$, and

$$
W_{1}(\varphi(\xi))=V_{1}(\xi),
$$

where $\varphi$ is defined by

$$
\varphi(\xi)=\int_{0}^{\xi} D\left(V_{1}(s)\right) d s .
$$

If $\int_{0}^{\infty} D\left(V_{1}(s)\right) d s=C_{1}<\infty$ or $\int_{0}^{-\infty} D\left(V_{1}(s)\right) d s=C_{2}>-\infty$, then $W_{1}(\xi)=0$ for $\xi \geqslant C_{1}\left(\right.$ resp. $W_{1}(\xi)=1$ for $\left.\xi \leqslant C_{2}\right)$.

2. Proof of the Theorem. We recall that $\left(1^{\prime}\right)$ can be converted into the first order system

$$
q^{\prime}(\xi)=p(\xi), \quad p^{\prime}(\xi)=-c p(\xi)-f(q(\xi)) .
$$


Therefore, the slope of a trajectory $V$ in the $(q, p)$ phase plane will satisfy

$$
d p / d q=-c-f(q) / p
$$

(cf. [2]).

Proof OF (iv) $\Rightarrow$ (iii). $W_{2}$ satisfies

$$
\left(D\left(W_{2}\right) \cdot W_{2}^{\prime}\right)^{\prime}+c \cdot W_{2}^{\prime}=-\left(k\left(W_{2}\right)\right)^{\prime},
$$

which can be written as

$$
D\left(W_{2}\right) \cdot W_{2}^{\prime}+c \cdot W_{2}=-k\left(W_{2}\right)
$$

and

$$
\left(D\left(W_{2}\right) \cdot W_{2}^{\prime}\right)^{\prime}+c W_{2}^{\prime}=-k^{\prime}\left(W_{2}\right) \cdot W_{2}^{\prime} .
$$

Solving (13) for $W_{2}^{\prime}$ and inserting into (14) then gives (in the sense of distributions on $\left\{0<W_{2}<1\right\}$ )

$$
\left(D\left(W_{2}\right) \cdot W_{2}^{\prime}\right)^{\prime}+c \cdot W_{2}^{\prime}=\frac{k\left(W_{2}\right)}{D\left(W_{2}\right) \cdot\left(c W_{2}+k\left(W_{2}\right)\right)}=\frac{-f\left(W_{2}\right)}{D\left(W_{2}\right)}=-g\left(W_{2}\right),
$$

i.e. $W_{2}$ satisfies $\left(2^{\prime}\right)$ on $\left\{\xi \mid 0<W_{2}(\xi)<1\right\}$. Trivially, (15) and, hence, $\left(2^{\prime}\right)$ will hold in the interior of the sets $\left\{W_{2}=0\right\},\left\{W_{2}=1\right\}$. At the boundary point of $\left\{0<W_{2}<\right.$ $1\}$, it suffices to note that (13) implies that $D\left(W_{2}\right) \cdot W_{2}^{\prime}$ is still absolutely continuous across the boundary; hence $\left(2^{\prime}\right)$ holds everywhere. We have found a solution $W_{2}$ of $\left(2^{\prime}\right)$; its uniqueness follows from the normalizations imposed on $W_{2}$ and from the smoothness properties of $D$ and $g$; thus $W_{1}=W_{2}$.

Proof OF (ii) $\Rightarrow$ (i). Similar.

Proof of (iii) $\Rightarrow$ (iv). Let $W_{1}$ be a solution of $\left(2^{\prime}\right)$. Rewrite $\left(2^{\prime}\right)$ on $\left\{0<W_{1}<1\right\}$ as the system

$$
W_{1}^{\prime}(\xi)=\frac{-c \cdot W_{1}(\xi)-\tilde{k}(\xi)}{D\left(W_{1}(\xi)\right)}, \quad \tilde{k}^{\prime}(\xi)=g\left(W_{1}(\xi)\right)
$$

or alternatively as

$$
W_{1}^{\prime}(\xi)=\frac{\tilde{P}(\xi)}{D\left(W_{1}(\xi)\right)}, \quad \tilde{P}^{\prime}(\xi)=-c \cdot \frac{\tilde{P}(\xi)}{D\left(W_{1}(\xi)\right)}-g\left(W_{1}(\xi)\right)
$$

$\left(\tilde{P}=-c W_{1}-\tilde{k}\right)$. It follows as in $[2$ or 4$]$ that $W_{1}$ is strictly monotone on $\left\{0<W_{1}\right.$ $<1$; ; hence, we can define

$$
k(r)=\tilde{k}(\xi) \text { if } W_{1}(\xi)=r
$$

As in [2], we conclude that

$$
k^{\prime}(r)=\frac{-g(r) \cdot D(r)}{c \cdot r+k(r)}=\frac{-f(r)}{c r+k(r)}
$$

for $0<r<1$. It follows that $\left(2^{\prime \prime}\right)$ holds on $\left\{0<W_{2}<1\right\}$. Also, $\left(2^{\prime}\right)$ implies that $D\left(W_{1}\right) \cdot W_{1}^{\prime}$ is absolutely continuous everywhere; hence (16) and (18) imply that

$$
k(r) \rightarrow 0 \quad \text { as } r \rightarrow 0, \quad k(r) \rightarrow-c \quad \text { as } r \rightarrow 1,
$$


and (since $W_{1}^{\prime}>0$ a.e.) $k(r)>c r$ for $0<r<1$. The same argument $\left(D\left(W_{1}\right) \cdot W_{1}^{\prime}\right.$ is absolutely continuous) then shows that (2") holds, in fact, across the boundary points of $\left\{0<W_{1}<1\right\}$, hence for all $\xi \in \mathbf{R}$.

ProOF OF (i) $\Rightarrow$ (ii). Similar.

Proofs of the Transformation Formula and (ii) $\Rightarrow$ (iv). The function $\varphi$ is well defined on some interval; its inverse $\psi=\varphi^{-1}$ satisfies (on the interior of the range of $\varphi)$

$$
\psi^{\prime}(\xi)=1 / D\left(V_{1}(\psi(\xi))\right)
$$

Thus, letting $\tilde{W}(\xi)=V_{1}(\psi(\xi))$, we obtain

$$
\tilde{W}^{\prime}(\xi)=V_{1}^{\prime}(\psi(\xi)) \cdot \frac{1}{D\left(V_{1}(\psi(\xi))\right)}=\frac{1}{D(\tilde{W}(\xi))} \cdot(-c \tilde{W}(\xi)-k(\tilde{W}(\xi)))
$$

Therefore, $\tilde{W}$ satisfies (2") (in the sense of distributions) in the set

$$
M=\left(\int_{0}^{-\infty} D\left(V_{1}(s)\right) d s, \int_{0}^{\infty} D\left(V_{1}(s)\right) d s\right) .
$$

If this set is the whole real line, we are done, since then $\tilde{W}=W_{2}$. Otherwise, continue to let $\tilde{W}$ be 0 (resp. 1) outside $M$ and note that (21) and this construction imply that $D(\tilde{W}) \cdot \tilde{W}^{\prime}$ is absolutely continuous on all of $\mathbf{R}$. Thus (2") holds on $\mathbf{R}$ also in this case.

Proof OF (iii) $\Rightarrow$ (i). Rewrite the equation for $W_{1}$ as in (16) and (17) to obtain a solution $k$ of (19) with the properties listed in (7). Using $P(r)=-c \cdot r-k(r)$ and the arguments of [2], it follows that a solution $V_{1}$ of $\left(1^{\prime}\right)$ exists. The proof of the Theorem is complete.

3. Consequences and remarks. To illustrate the result, we start with an

ExAmple. Take $f(u)=\frac{1}{2} u^{2} \cdot(1-u)$ in (1). Then (1) becomes "Huxley's equation" (up to the factor $\frac{1}{2}$ ), and for the speed $c=\frac{1}{2}$ an explicit travelling wave solution is known:

$$
V_{1}(\xi)=1 /\left[1+\exp \left(\frac{1}{2} \xi\right)\right] .
$$

This function can also be obtained as a wave solution of $\left(1^{\prime \prime}\right)$ if we choose $k(r)=-\frac{1}{2} \cdot r^{2}$. With this choice of $k,\left(1^{\prime \prime}\right)$ is known as "Burger's equation" with viscosity [9]. Finally, for $D(u)=u, g(u)=\frac{1}{2} u \cdot(1-u)$, we find the solution

$$
W_{1}(\xi)=\left[1-\exp \left(\frac{1}{2} \xi-\log 2\right)\right]_{+}
$$

for the same speed, which can be obtained by using the transformation (8) or, more easily, by directly integrating ( 2 "). In this case (2) becomes "Fisher's equation with density-dependent diffusion" up to the factor $\frac{1}{2}$. The special solution (23) has been given previously in $[1,3,7,8]$.

More generally, the existence results for travelling wave solutions in [3] (case $\left.D(u)=u^{n}, g(u)=u \cdot(1-u)\right)$ and in [1] $\left(D(u)=u^{n}, g(u)=u \cdot(1-u)\right.$ or $g(u)$ $=u \cdot(1-u) \cdot(u-a))$ can be reduced to the very general existence theory in [2] that is concerned with problem (1) but uses the properties of equation (7) for $k$ quite extensively. In particular, the phenomenon that there exists a unique wave speed $c^{*}$, 
if in (2) $g(r)<0$ for all small $r$, and an interval $\left[c^{*}, \infty\right)$, of possible speeds, if $g(r)>0$ for all $r$, will persist for quite general choices of $D$. Also, similar results follow naturally if $D(u)=u^{n}$ with $n<0$.

The above example suggests that one can find explicit solutions for (2) if $D(u)=u^{n}$ and $g(u)=\frac{1}{2} u^{m} \cdot(1-u)$, if $n+m=2$, since in all these cases $k(r)=$ $-\frac{1}{2} r^{2}$ is the solution of (7). This class of solutions has been studied in [7]. Also, by making other choices of $k(\cdot)$, new special solutions of equations of types (1) or (2) can be generated, choosing $c$ and

$$
f(r)=-k^{\prime}(r) \cdot(c r+k(r))
$$

(resp.

$$
\left.g(r)=\frac{-k^{\prime}(r)}{D(r)} \cdot(c r+k(r))\right) .
$$

Such solutions could be useful as sub- or supersolutions of (1) (resp. (2)).

Examining the proof of the Theorem, one sees that the conclusions (without the uniqueness assertions) and the transformation formula still hold if $f, g, D$ are only, say, piecewise continuous, and $f$ and $g$ are bounded. This allows one to deduce the existence of solutions also for these more general equations. Also, unbounded $f, g, D$ can be allowed; for the sake of brevity, these generalizations have been omitted.

Finally, it should be pointed out that equivalences of the form (i) $\Leftrightarrow$ (ii) also hold for equations of the form

$$
w_{t}-b\left(w_{x}\right)_{x}=h(w),
$$

if $b$ is, say, continuous and strictly increasing: A wave solution of (26) with speed $c>0$ is also a wave solution of

$$
w_{t}-b\left(w_{x}\right)_{x}=\tilde{k}(w)_{x}
$$

if

$$
\tilde{k}^{\prime}(r) \cdot b^{-1}(-c: r-\tilde{k}(r))=h(r), \quad 0<r<1,
$$

where $b^{-1}$ denotes the inverse of $b$. These types of equations (with $b(r)=r^{q}$, $h(r)=r \cdot(1-r))$ have been discussed in [3]. With the equivalence (26) $\Leftrightarrow\left(26^{\prime}\right)$, (27), one can reduce this problem to a phase plane discussion in the spirit of [2] and an integration of a first order equation.

Note ADDED IN PROOF. After this note had been accepted for publication, the author learned that a version of the transformation (8), (9) has previously been employed in [10] to the case " $D(r)>0$ for $0 \leqslant r \leqslant 1$ ".

\section{REFERENCES}

1. D. G. Aronson, Density-dependent interaction-diffusion systems, Proc. Adv. Sem. on Dynamics and Modeling of Reactive Systems (Madison, 1979), Academic Press, New York, 1980.

2. D. G. Aronson and H. F. Weinberger, Multidimensional nonlinear diffusion arising in population genetics, Adv. in Math. 30 (1978), 33-76.

3. C. Atkinson, G. E. H. Reuter and C. J. Ridler-Rowe, Travelling wave solutions for some nonlinear diffusion equations, SIAM J. Math. Anal. 12 (1981), 880-892.

4. P. C. Fife, Mathematical aspects of reacting and diffusing systems, Springer-Verlag, Berlin and New York, 1979. 
5. W. S. C. Gurney and R. M. Nisbet, The regulation of inhomogeneous populations, J. Theoret. Biol. 52 (1975), 441-457.

6. M. E. Gurtin and R. C. MacCamy, On the diffusion of biological populations, Math. Biosci. 33 (1977), $35-49$.

7. W. I. Newman, Some exact solutions to a non-linear diffusion problem in population genetics and combustion, J. Theoret. Biol. 85 (1980), 325-334.

8. W. I. Newman and C. Sagan, Galactic civilizations: population dynamics and interstellar diffusion, Icarus 46 (1981), 293-327.

9. J. Smoller, Shock waves and reaction diffusion equations, Springer-Verlag, Berlin and New York, 1982.

10. K. P. Hadeler, Free boundary value problems in biological models, Free Boundary Problems: Theory and Applications (A. Fasano and M. Primicerio, eds.), vol. II, Pitman, Boston, 1983, pp. 664-671.

Department of Mathematics, University of TeXas, Austin, TeXas 78712

Current address: Department of Mathematics, Georgetown University, Washington, D.C. 20057 